\title{
Settling of Spherical Objects Through Thixotropic Fluids; A Statistical Approach
}

\author{
Nahid Maleki-Jirsaraei ${ }^{1}$, Samira Hasani ${ }^{1} \&$ Somayeh Azizi $^{1}$ \\ ${ }^{1}$ Complex Systems Laboratory, Physics Department, Alzahra University, Tehran, Iran \\ Correspondence: Nahid Maleki-Jirsarei, Complex Systems Laboratory, Physics Department, Alzahra University, \\ P.O. Box 1993891176, Tehran, Iran. E-mail: maleki@alzahra.ac.ir
}

\author{
Received: April 3, $2018 \quad$ Accepted: April 10, $2018 \quad$ Online Published: April 28, 2018 \\ doi:10.5539/mas.v12n5p72 URL: https://doi.org/10.5539/mas.v12n5p72
}

\begin{abstract}
The falling of an object through a non-Newtonian fluid is an interesting problem, depending on the details of the rheology of the fluid. In this paper we report on the settling of spherical objects through non-Newtonian fluids: Laponite. A falling object's behavior in passing through a thixotropic colloidal suspension of synthetic clay, Laponite, has been reported to have many behavioral regimes. Here we report a new regime where irregular motion is observed. We argue that this irregular motion may be interpreted as onset of chaos. Observation of this regime depends on the size of the falling sphere, relaxation time of fluid and concentration of particles in the suspension.
\end{abstract}

Keywords: non-newtonian fluids, laponite, thixotropic, oscillating transient, drag force

\section{Introduction}

The falling of an object through a Newtonian fluid is a classical problem in fluid mechanics. The drag force experienced by a falling ball of radius $R$, when moving with terminal velocity $U$, through a fluid with viscosity $\mu$ is $\mathrm{F}_{\mathrm{D}}=6 \pi \mu \mathrm{RU}$ [1]. Though fundamentally correct, this formula has been adjusted through modern considerations [2, 3, 4, and 5] and forms the basis of measuring viscosity in a Newtonian fluid. In recent years, the settling of a solid object through a non-Newtonian fluid has received attention [6,7, and 8]; due to various rheological and structural properties of non-Newtonian fluids, the problem of a settling sphere in non-Newtonian fluid is less known. A falling sphere in a non-Newtonian fluid behaves in a complex fashion, in short term it does not always reach a terminal velocity [9], though it may come to a complete rest, sometimes it approaches a terminal velocity, after an oscillating transient [7]. In this paper we present evidence that a sphere falling in Laponite may experience an irregular even chaotic motion. We have performed experiments in Laponite (B, Laponite is smectite hectorite clay. The Laponite particle has a disk shape typically with diameter of $25 \mathrm{~nm}$ and thickness of $0.92 \mathrm{~nm}$. There are different grades of Laponite for a complete listing see [10].

\section{Experiment Setup}

Laponite is a synthetic clay frequently used as a model aging material which is a thixotropic fluid $(\rho \mathrm{l}=1109 \mathrm{~kg} / \mathrm{m} 3)$ [11]. Figure 1 shows shear stress vs. shear rate and figure 2 shows viscosity vs. shear rate for Laponite. The Laponite powder is mixed slowly with swirling distilled water approximately at PH 8 for 1 hour with mechanical stirrer [Heidolph RZR 2041] at $500 \mathrm{rpm}$. For 10 days, the fluid is stirred every 2 days for 1 hour at $500 \mathrm{rpm}$. Just before the beginning of the experiment the fluid is mixed (1 hour at $500 \mathrm{rpm}$ ) in order to obtain reproducible initial state. We used Laponite suspension with concentration $1.5 \mathrm{wt} \%$, and experiments were repeated with different resting times. This concentration is the lowest concentration for which Laponite stays a Gel-like for the resting times we have experimented with, lower concentrations one may run into liquid regime [12]. Selected steel sphere did not move in Laponite of concentration $3 w t \%$. Prepared fluids were put into a cylindrical vessel with a depth of $45 \mathrm{~cm}$ and an inner diameter of $6 \mathrm{~cm}(\mathrm{D})$, the fluid depth is $40 \mathrm{~cm}$ into the cylinder. Laponite was left at rest a given time during which it could restructure so that its apparent viscosity increased. Then a steel sphere was put with pliers in the fluid just below the free surface and closes to the central axis of the vessel and let go. The settling sphere process is recorded with a CCD camera. All motions are observed at low Reynolds number ( $\operatorname{Re}<10$ ), where flow is laminar [1]. We used steel spheres in different sizes $(2.5 \mathrm{~mm}<\mathrm{d}<14 \mathrm{~mm})$ and same density $(\rho \mathrm{s}=7753 \mathrm{~kg} / \mathrm{m} 3)$. The ratio of sphere to vessel radius $\left(0.04<\frac{\mathrm{d}}{\mathrm{D}}<\right.$ $0.2)$ is small, so wall effects are negligible $[13,18]$. 


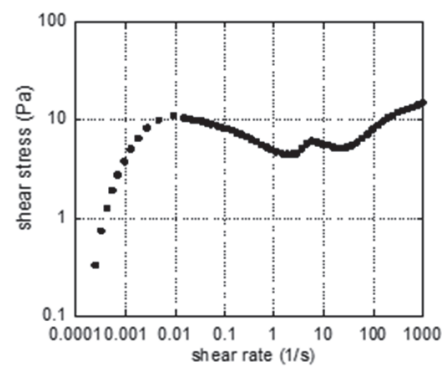

Figure 1. Shear stress vs. shear rate for Gel and Laponite (1.5wt \%)

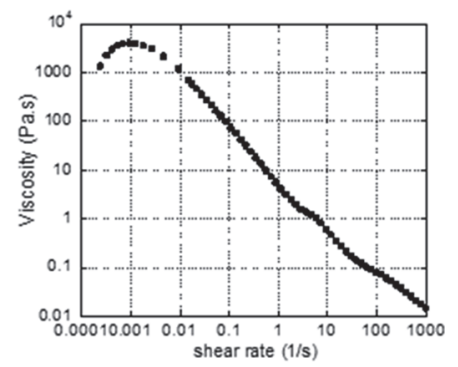

Figure 2. Viscosity vs. shear rate for Gel and Laponite (1.5wt \%)

\section{Observations}

For a sphere object settles through the Laponite suspension, literatures explained three regimes for its motion. For short times of rest, settling spheres move through the Laponite always at constant, large velocity (regime 1). For intermediate times of rest, spheres move at intermediate velocity which continuously decreases (regime 2). And for long times of rest, they move at very low velocity (regime 3) [14,19]. We observed in our experiments, other than three regimes that are explained above, there is another regime for settling object through the Laponite. When small steel spheres (their diameters are $3 \mathrm{~mm}$ and $4 \mathrm{~mm}$ ) are selected and experiments are done at various times of rest (tw), settling sphere through the Laponite does not always move at a constant velocity or acceleration. There are oscillations at velocity of settling object in different times of rest (figure 5). Time of rest and size of balls are important factors. Also small object falling through Laponite does not move on a straight line to reach the vessel bottom $[15,20]$. Additional experiments were done on various diameters of steel spheres: $1.5,2,2.5,3,3.5,4$ and $4.5 \mathrm{~mm}$. For small spheres with diameter less than $2.5 \mathrm{~mm}$, balls move slowly or they don't move through the fluid at all (regime 3). Spheres with diameters between 2.5 and 3.5, balls do not move with constant velocity and have oscillatory motion. Spheres with diameters bigger than $3.5 \mathrm{~mm}$ move with large constant velocity through the fluid (regime 1). These results were achieved for small time of rest (10 minutes). Figure 3 shows experiments done in different times of rest. More experiments were done between two glass walls. There wall effect is too large but small falling spheres don't move with constant velocity.

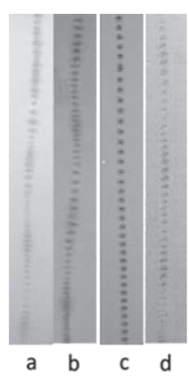

Figure 3. Set of video images of steel sphere falling through Laponite $1.5 \% \mathrm{wt}$.For the sake of distinguishable images time interval is different for each image. (a) $3 \mathrm{~mm}$ diameter steel sphere at $\mathrm{tw}=30$ minute and time interval is $1.6 \mathrm{~s}$ (b) $3 \mathrm{~mm}$ diameter steel sphere at $\mathrm{tw}=10$ minute and time interval is $0.4 \mathrm{~s}$. (c) $4 \mathrm{~mm}$ diameter steel sphere at tw $=10$ minute, and time interval is $0.12 \mathrm{~s}(\mathrm{~d}) 4 \mathrm{~mm}$ diameter steel sphere at $\mathrm{tw}=30$ minute and time interval is $0.4 \mathrm{~s}$. 


\section{Analysis}

Distance of settling sphere from free surface and its velocity is computed using motion analyzer and commercially available software. Graphs for velocity of sphere falling through the Laponite suspension as a function of time are shown in Figures 4, which show irregular motion of a falling spherical object through Laponite suspension.
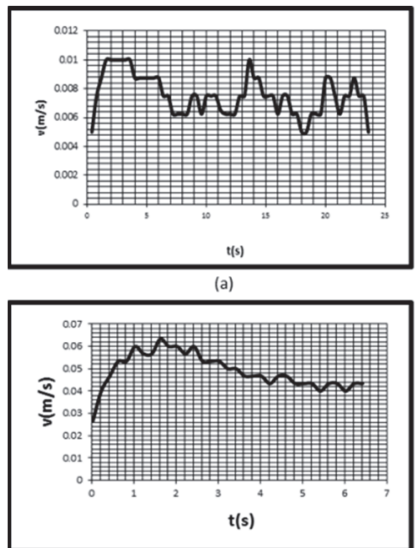

(c)

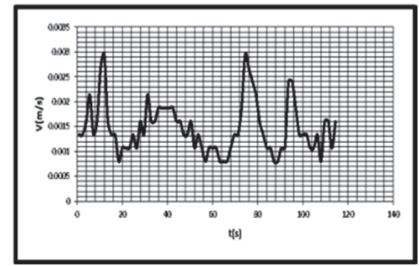

(b)

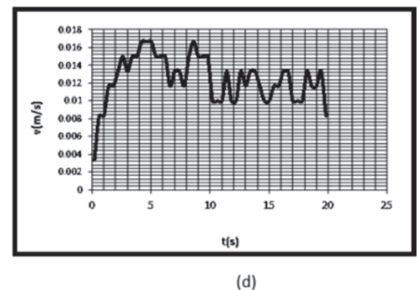

Figure 4. Velocity as a function of time for falling spheres in Laponite ,a) $3 \mathrm{~mm}$ diameter sphere, $t \mathrm{w}=10 \mathrm{~min}$, and b) $3 \mathrm{~mm}$ diameter sphere, $\mathrm{tw}=30 \mathrm{~min}, \mathrm{c}) 4 \mathrm{~mm}$ diameter sphere, $\mathrm{tw}=10 \mathrm{~min}$, and d) $4 \mathrm{~mm}$ diameter sphere, $\mathrm{tw}=30 \mathrm{~min}$

To describe the motion of the falling sphere through Laponite we appeal to the "House of Cards" structure, perhaps the ball is falling in stages, rather like a fruit through a tree. Let us assume that the velocity of the falling object is vi at the end of a suitable interval. Velocity at the end of the next interval $\mathrm{Vi}+1$ is related to the previous interval is related to the velocity of the previous interval and the innate properties of the fluid. Previous work shows that exert forces on sphere falling through non-Newtonian fluid may be functions of $v_{i}^{2}$ and $v_{i}$ [2-8]. So we consider iterative processes of the form

$$
v_{i+1}=f\left(v_{i}\right)=a v_{i}^{2}+b v_{i}+c
$$

Here we have assumed a quadratic $f\left(v_{i}\right)$ whereas more complex functions may be taken. Figure 5 shows $v_{i+1}$ versus $\mathrm{v}_{\mathrm{i}}$ for different falling spheresIn The difference between the cases is due to the structure of Laponite. Difference between coefficients of square velocity in fitting functions of above cases shows differences in drag forces. Drag force is related to flow induced structure. When this structures breaks, sphere suddenly accelerates [9]. So Figure 5 shows three types of attractors for different spheres falling through Laponite suspension.
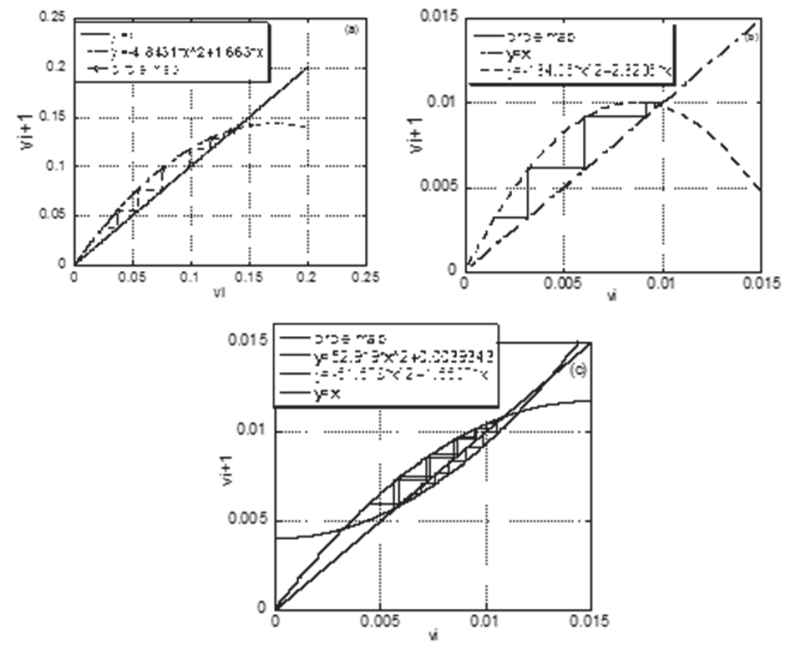

Figure 5. Diagram displaysVi+1 (vertical axes) versus Vi (horizontal axes) for spheres, this figure shows three 
different behavior of sphere through Laponite suspension a)This graph shows the simple fixed-point attractive behavior for falling sphere with $\mathrm{D}=4 \mathrm{~mm}$ and aged time of laponite is tw=10min. $\mathrm{b}$ ) this graph shows the orbit settles into a two-cycle for falling sphere with $D=4 \mathrm{~mm}$ and aged time of laponite is tw=30min. $\mathrm{c}$ ) this graph shows the orbit settles into a 26 -cycle for falling sphere with $\mathrm{D}=3 \mathrm{~mm}$ and aged time of laponite is $\mathrm{tw}=10 \mathrm{~min}$

\section{Time Scale from Viscosity to Modules}

The other point is the relative timescale of velocity fluctuations and the relaxation time. The Deborah number De, is the ratio of relaxation time of the fluid $(\tau)$ to time scale of process $\left(t_{a}\right)$. This dimensionless number is significant for viscoelastic fluids. A large value of De $(>1)$ denotes a dominantly elastic response and $\mathrm{De}<1$ indicates viscous behavior [16]. We believe that for any cycle of falling and rising sphere velocity, transition between viscous and elastic behaviors happen. And transition happens when $\mathrm{De}=1$ therefore $\tau=\mathrm{t}_{\mathrm{a}}$. Figure 6 shows loss and storage modules and relaxation time versus elapsed time for prepared Laponite suspension. We have used an oscillatory experiment (amplitude oscillatory $(\gamma 0)=1 \%$ and frequency $0.1 \mathrm{~Hz}$ ) to estimate the both modulus. And relaxation time are calculated by $\tau=G^{\prime} / \omega G^{\prime \prime}$ [17] where $G^{\prime}, G^{\prime \prime}$ are storage and loss modulus respectively. Relaxation time increases with time and it obeys a power law $\tau \sim \mathrm{t}_{\mathrm{w}}{ }^{0.2}$, so when $\mathrm{t}_{\mathrm{a}} \sim \mathrm{t}_{\mathrm{w}}{ }^{0.2}$, transition happens. Transition between viscous and elastic behavior is more substantial when small sphere $(D=3 \mathrm{~mm})$ falls through aged Laponite suspension $(\mathrm{tw}=10 \mathrm{~min})$. Comparison between time scale process that obtains from both experiments (above oscillatory Rheology measurement and free fall of spheres through Laponite suspension) shows that they have same order.

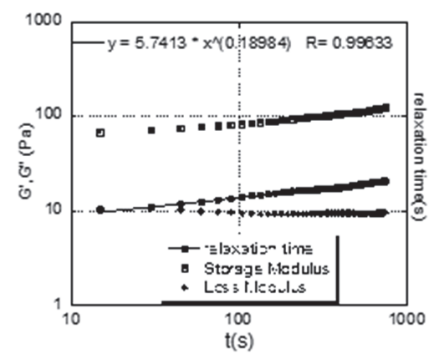

Figure 6. Shows storage modulus $\left(\mathrm{G}^{\prime}\right)$, loss modulus $\left(\mathrm{G}^{\prime \prime}\right)$ and relaxation time as a function of time (frequency= $0.1 \mathrm{~Hz}, \gamma 0=1 \%)$.thick red line represents a power low fit to relaxation time - time data $\left(\tau \sim \mathrm{t}_{\mathrm{w}}{ }^{0.2}\right)$

\section{Concluding Remarks}

We have presented experimentally various behaviors of spheres falling through Laponite. Experiments of spheres falling through colloidal suspension of synthetic clay Laponite show four different regimes. Three of these regimes have been discussed before, in this paper we present a fourth regime showing irregular perhaps chaotic motion. The onset of this regime mostly depends on the size of the falling sphere and aging of Laponite suspension and concentration of particles. Difference between coefficients of square velocity in fitting functions of above cases (Figure 5) shows differences in drag forces. Drag force is related to flow induced structure. When this structures breaks, sphere suddenly accelerates. We found that for oscillatory cases we may have a chaotic phenomenon. It also seems that for any cycle of falling and rising sphere's speed, there is a transition between viscous and elastic behavior. This transition happens when De $\sim 1$, our results showed that the time scale of these speed fluctuations and relaxation time of Laponite suspension are comparable.

\section{Acknowledgments}

We would like to thank Professor Daniel Bonn and Professor Shahin Rouhani for their collaboration. This work has been supported by the Center of International Scientific Studies \& Collaboration (CISSC). We appreciate member of the Complex Systems Laboratory of Al-Zahra University for their cooperation.

\section{References}

Abaid, N., Adalsteinsson, D., Agyapong, A., McLaughlin, R. M. (2004). Physics of Fluids, 16, 1567-1580.

Arigo, M. T., \& McKinley, G. H. (1998). Rheol. Acta., 37, 307-327.

Asima, S., Yogesh, M. J., \& Ashutosh, S. (2009). Ind. Eng. Chem. Res., 48, 8211-8218. 
Bonn, D., Tanase, S., Abou, B., Tanaka, H., \&Meunier, J. (2002). Phys. Rev. Lett., 89, 015701.

Cummins, H. Z. (2007). Journal of Non-Crystalline Solids, 353, 3891-3905.

Faber, T. E. (1995). Fluid dynamics for physicists. Cambridge University Press, United Kingdom.

Ferroir, T., Huynh, H. T., Chateau, X., \& Coussot, P. (2004). Physics of fluid, 16, 1070-6631.

Geslin, B., talini, L., Herzhhaft, B., peysson, Y., \& Allain, C. (2006). Phys. Rev. E 74, 04250

Gueslin, B. (2006). Physics of Fluids, 18, 103101.

Hervé, T. (2007). Journal of Rheology, 51, 125.

Jayaraman, A., \& Belmonte, A. (2003). Phys. Rev. E., 67, 65301.

Joshi, Y. M., Reddy, G. R., \& Kulkarni, L. A. (2008). Proc. R. Soc. A, 464, 469-489.

Lin, B., Yu, J., \& Rice, S. A. (2000). Phys. Rev. E 62, 3.

Oliver, G., \& Harlen, J. (2002). Non-Newtonian Fluid Mech., 108, 411-430.

Renganathan, K., Turton, R., \& Clark, N. N. (1989). Powder Technology, 58, 279-284.

Richardson, E. G (1948). Proc. of the Phys. Soc., 61, 352-367.

Stefan von Kann, Jacco H. Snoeijer, \& Devaraj van der Meer. (2013). PHYSICAL REVIEW, E 87, 042301.

Tabuteau, H., Coussot, P., de Bruyn, J. R., \& Rheol, J. (2007). 51(1), 125-137.

White, F. M. (2003). Fluid Mechanics. McGraw-Hill Higher Education.

\section{Copyrights}

Copyright for this article is retained by the author(s), with first publication rights granted to the journal.

This is an open-access article distributed under the terms and conditions of the Creative Commons Attribution license (http://creativecommons.org/licenses/by/4.0/). 